\title{
Standardization of Power System Protection Settings Using IEC 61850 for Improved Interoperability
}

\author{
Qiteng Hong, Steven M. Blair, Victoria M. Catterson, \\ Adam Dyśko, Campbell D. Booth \\ Institute for Energy and Environment \\ University of Strathclyde \\ Glasgow, G1 1XW, UK \\ q.hong@strath.ac.uk
}

\author{
Tahasin Rahman \\ National Grid \\ Warwick, CV34 6DA, UK \\ tahasin.rahman@nationalgrid.com
}

\begin{abstract}
One of the potential benefits of smart grid development is that data becomes more open and available for use by multiple applications. Many existing protection relays use proprietary formats for storing protection settings. This paper proposes to apply the IEC 61850 data model and System Configuration description Language (SCL), which are formally defined, to represent protection settings. Protection setting files in proprietary formats are parsed using rule-based reasoning, mapped to the IEC 61850 data model, and exported as SCL files.
\end{abstract}

An important application of using SCL-based protection setting files is to achieve protection setting interoperability, which could bring multiple compelling benefits, such as significantly streamlining the IED configuration process and releasing utilities from being "locked in" to one particular vendor. For this purpose, this paper proposes a uniform configuration process for future IEDs. The challenges involved in the implementation of the proposed approach are discussed and possible solutions are presented.

Index Terms-- IEC 61850, power system protection, smart grid.

\section{INTRODUCTION}

A key assumption about the smart grid is that system data will be available for a wide variety of automated applications. However, critical data such as protection settings are generally recorded in proprietary formats or unstructured text files. Often, such text files have been created manually in the past, and formats differ significantly between relays and vendors. Therefore, it is difficult for automated systems to manipulate these data directly.

This paper proposes to use the data model provided by the IEC 61850 standard to represent protection settings. The setting information is parsed from the original setting text files and mapped to the IEC 61850 data model. The text files are exported subsequently in System Configuration description Language (SCL) [1] format which is formally defined. The SCL-based protection setting files are ready for multiple applications, such as: importing into a protection simulation system to validate protection operation [2]; a protection setting error detection system, as described in [3]; integration with a smart grid data management system; and for enforcing protection setting interoperability. Among these applications, this paper will focus on applying the IEC 61850-based data model to achieve protection setting interoperability.

The IEC 61850 standard aims, among other benefits, to provide interoperability between Intelligent Electronic Devices (IEDs). However, IEC 61850 does not provide interoperability for protection settings. At the moment, the configuration process for each IED is complex. IED protection settings are different for each vendor device, and proprietary software or communications protocols are necessary. The engineers need to be trained to use different tools for the same purpose. Furthermore, because protection setting interoperability has not yet been achieved, replacing one vendor's relay with another can be very time-consuming because the engineer has to manually convert all settings. This is particularly problematic for legacy devices.

This paper will introduce how the original protection setting files are converted to SCL-based files and how to use this approach to achieve protection setting interoperability. The paper outlines the background to the research (Section II), and the existing IEC 61850 data model (Section III). Section IV introduces the process of parsing the information from original files, mapping to IEC 61850 data model, and exporting to an SCL file. A proposed IED configuration process to achieve protection setting interoperability is presented in Section V. The potential benefits and challenges - and possible solutions to these challenges - are discussed along with ongoing and future work.

\section{RESEARCH CONTEXT}

The work presented in this paper is part of the research as described in [3] which proposes a rule-based intelligent system to detect hidden errors in protection settings.

Due to the growth of network complexity, solely relying on personnel and procedures to assess the validity of the protection relay settings may occasionally leave errors undetected until a failure is experienced, which may lead to power outage or other severe consequences. The system 
proposed in [3] automates the setting verification process and aims to increase the reliability of the validating results. The use of the standardized approach described in this paper simplifies the task of developing and maintaining the validation system.

\section{EXISTING IEC 61850 DATA MODEL}

IEC 61850 is an international standard for power system automation and communications [4]. The implementation of this standard enables communications interoperability among IEDs from various vendors. To achieve this interoperability, IEC 61850 provides a data model which is formally defined [5, 6]. The data model decomposes the application functions into the smallest entities called logical nodes (e.g., a virtual representation of a circuit breaker). Multiple logical nodes build a logical device (e.g. a multi-function protection device in an IED) and multiple logical devices then compose a physical device (e.g., a physical IED).

The logical nodes are grouped according to the functions they perform. For protection functions, the logical node group is Group P, which contains 30 logical node definitions. Fig. 1 shows the logical node PDIS, which describes distance protection functions. Each distance protection zone is modeled as an instance of the PDIS class. Within the logical node, there are data objects related to the settings of the protection function, e.g., PoRch represents the polar reach (diameter of the Mho diagram) while DirMod represents directional mode of the protection zone. PoRch and DirMod are examples of a set of common data class (CDCs) [7], which define the data object and attributes of those objects. The data attributes specify more details about the data objects, e.g., the magnitude, the maximum and minimum values, etc. The names of these logical nodes, data objects, and data attributes are defined in a standard way to achieve interoperability.

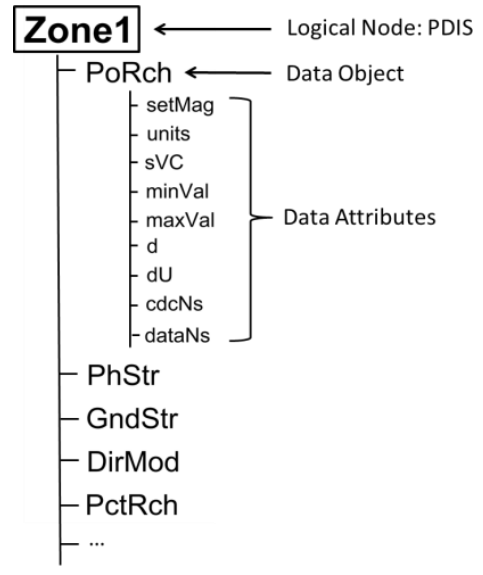

Fig. 1. PDIS logical node in a tree representation

\section{Representing Protection Settings Using The IEC 61850 DATA MODEL}

Given the variety of vendor-specific settings formats, a process has been developed by the authors to automatically translate protection settings to the IEC 61850 data model. The process was implemented in the object-oriented Java language which lends itself well to the data model defined in IEC 61850 , and which is platform independent.
The process, shown in Fig. 2, involves three steps:

1. Parse the vendor's original settings files into Java objects, which contain the setting parameters and their values.

2. Map the Java objects into a Java representation of the IEC 61850 data model (which will be referred to as IEC 61850 Java objects), i.e., map to logical nodes and CDC definitions.

3. Export the IEC 61850 Java objects to an SCL file.

Each step is described in more detail in the following sections.

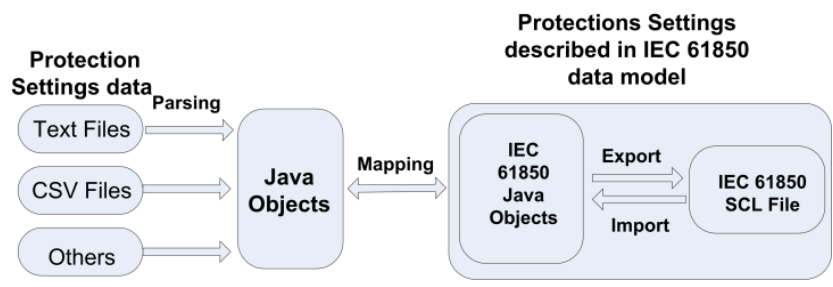

Fig. 2. Overall process of converting protection setting data into IEC 61850 format

\section{A. Parsing information from the original settings file}

Utility companies often use text files to record protection settings. This is because many legacy in-service relays cannot export their settings automatically. In order to manipulate the data contained in these text files, it is necessary to parse the setting data from the original files and assign appropriate values to Java variables.

There are several challenges involved in the parsing process:

1. There is no standard data representation for recording protection settings. Typically, each type of relay uses a unique data representation to record the settings within a text file.

2. For some legacy devices, there are no strict rules about what parameters and what information should be recorded.

3. Different names and different abbreviations could be used for the same parameter. Different units may also be used.

To address these problems, each relay type needs a specific parser to extract the information. The parser has to be highly adaptable to deal with various situations.

The authors propose to apply rule-based reasoning techniques for data parsing and data extraction purposes. This avoids many nested "if-then" statements, provides separation between rules and data, and can be combined with a larger expert system such as the one described in [3]. Other advantages of using rule-based reasoning are elaborated in [3].

The parsing process is illustrated in Fig. 3. The parser is composed of a set of rules for extracting data by applying regular expressions. A regular expression, with a general and powerful pattern-matching notation, allows the description and parsing of a segment of text with common characteristics [8]. Each rule contains a regular expression, which represents 
a certain string that contains a specific parameter in the original file. Each text file is imported line by line to the knowledge base, which matches the string to the rules' regular expressions. If matched, the rule will be executed to extract the data.

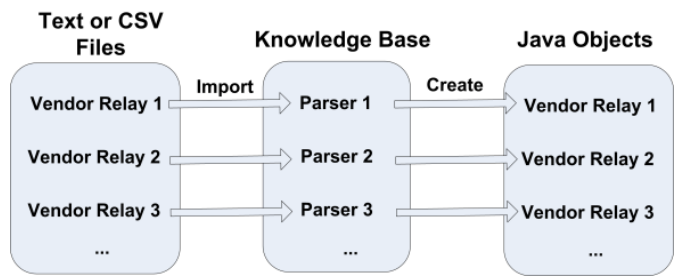

Fig. 3. Parsing protection setting information from text or CSV files

Fig. 4 shows a sample text file which records the settings of an Alstom P443 relay [9]. For a string which contains the Zone 1 reach and its direction, the parsing rule is illustrated in Fig. 5.

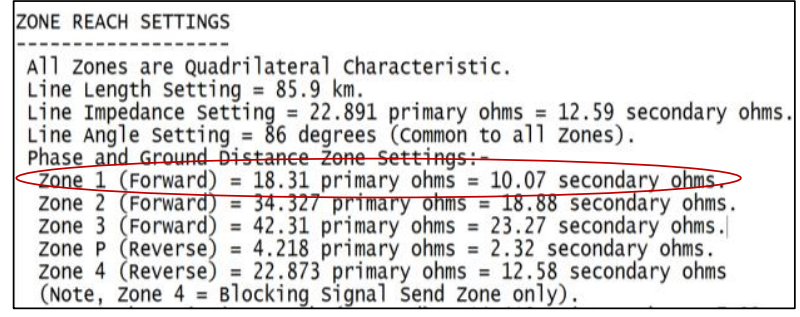

Fig. 4. Sample protection setting text file

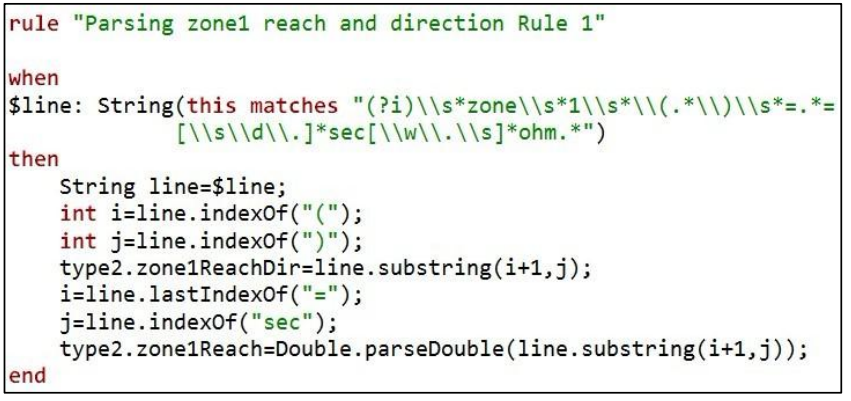

Fig. 5. Sample parsing rule for zone 1 reach and its direction

The "when" part defines the condition for executing the rule. The "then" part extracts the information and assigns the data to a Java object. "type2" is the name of a Java object created for this type of relay. "zone1Reach" and "zone1ReachDir" are attributes of the object which represent zone 1 reach and its direction, respectively. Since various presentations of the data exist, sometimes several rules are needed to parse one parameter. The number of rules applied largely depends on the setting parameters that a relay has. Typically, for legacy devices, around 50 rules are needed to parse all the information contained in the setting file.

For modern numerical relays, sometimes a vendor-specific file format is also used to store the settings, which can be exported as comma-separated values (CSV) files through the vendors' software tools. These CSV files can also be parsed into Java objects through the approach described above.

\section{B. Mapping settings to IEC 61850 defined Java objects}

To map the Java objects created in the parsing process to the IEC 61850 data model, a Java implementation of the IEC 61850 data model is needed. OpenIEC61850 [10] is an open source implementation of the IEC 61850 standards, which provides a Java-based IEC 61850 data model. The data model has been utilized by the authors to fully implement the CDCs and logical node classes defined in IEC 61850.

The mapping process is shown in Fig. 6. The lower rectangular area represents the Java objects created in the parsing process. Where possible, bi-directional mapping relationships between the original Java objects and the IEC 61850 Java objects are created, which not only allows the settings to be represented by IEC 61850 data models but also allows the settings in the IEC 61850 data model to be mapped to each individual relay settings. This makes the conversion of settings between two different vendors' relays possible, which brings significant convenience when replacing a relay. The use of a common representation is analogous to the approach used by the Common Information Model [11] for Energy Management System (EMS) data.

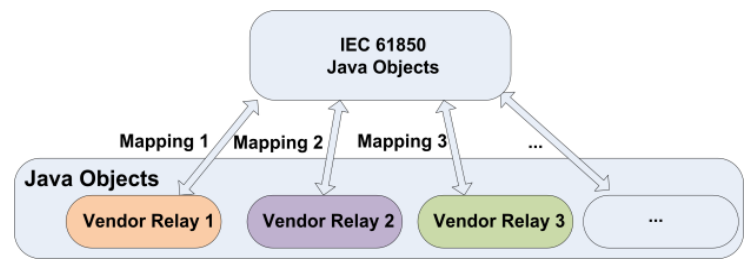

Fig. 6. Mapping to IEC 61850 Java objects

For example, as shown in Fig. 4, the Zone 1 reach is provided in ohms. In the IEC 61850 data model, the zone reach is represented by PctRch, which is Zone 1 reach expressed as a percentage of the protected line positive sequence impedance (Z1). The mappings between these two descriptions of Zone 1 reach are illustrated in Fig. 7.

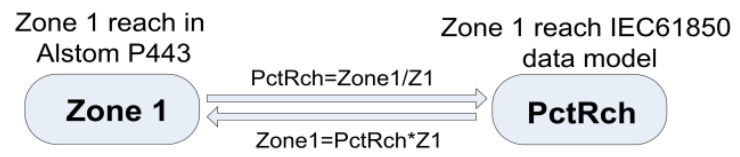

Fig. 7. Bi-directional mapping relationship of Zone 1 reach

Due to different capabilities of each relay, some information might be lost during the mapping process. In some cases, the mapping from the IEC 61850 data model to specific relay settings also might not be possible. This is illustrated in Fig. 8 for a Reyrolle THR-type high speed distance relay [12], where:

- $\mathrm{A}$ is the Zone 1 coarse setting,

- $\mathrm{G}$ is a number in percentage to fine adjust the coarse setting, and

- $E$ is the multiplier used to extend the zone reach when the protected line impedance is larger than maximum value of the coarse setting.

The Zone 1 reach is the product of these three parameters, i.e., $A \times G \times E$. Therefore, mapping the IEC 61850 data back 
to all these three parameters cannot be achieved without additional information or rules.

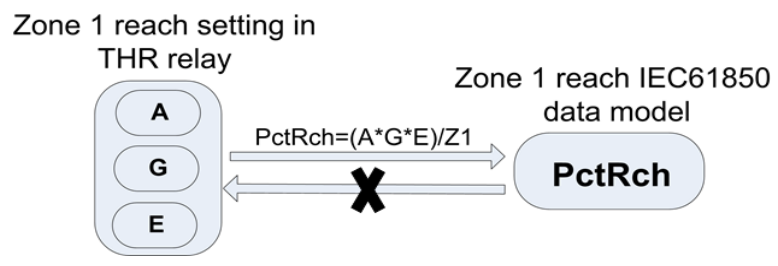

Fig. 8. One-way mapping relationship of Zone 1 reach

\section{Exporting the IEC 61850 Java objects to an SCL file}

Java objects only exist while the program is running, but it is essential to be able to save the data to a file for future use. IEC 61850 provides the SCL configuration language to describe IED capabilities and the interactions between IEDs [1]. The IEC 61850 Java objects can be exported to SCLformat files, which could be manipulated by software and used as a human-readable document.

An SCL file containing setting information could also be imported to create corresponding Java objects when necessary, e.g., for altering protection settings, or for error detection.

The exporting and importing process can be conveniently achieved using one of the existing software environments such as the Eclipse Modeling Framework [13], which provides a solution for conversion between Java objects and SCL files automatically, as described in [14].

\section{IMPROVING INTEROPERABILITY USING SCL-BASED PROTECTION SETTINGS}

\section{A. Existing IED configuration process}

The standard defines a process for IED configuration using SCL files, as shown in Fig. 9 [1]. Each IED has an IED Capability Description (ICD) file to tell the system configurator tool which logical nodes are available. By design, IEC 61850 does not define the internals of logical nodes.

Even following this process, there are still practical problems. The ICD files are created by IED configurator software or are provided by the vendors, and they only describe the IED capabilities without details about the protection settings. The setting process is still carried out inside the vendor-specific tools. Each vendor has a proprietary file format to describe the settings stored in the IED or communicated between the IED and the vendor software tool. Therefore, interoperability has not yet reached the protection settings level.

This configuration methodology has been adopted because different vendors implement different protection methods and technical features. Currently, IEC 61850 does not provide a full set of data objects which could describe all the technical specifications of relays from all vendors. Reference [15] raises some issues with the IEC 61850 data model, such as the lack of a comprehensive set of protection function parameters in the pre-defined logical nodes, and the ambiguous definition of mandatory and conditional data objects. Furthermore, vendors may prefer to keep their own setting file formats both as a business strategy and for developing new products without being constrained by a standard.

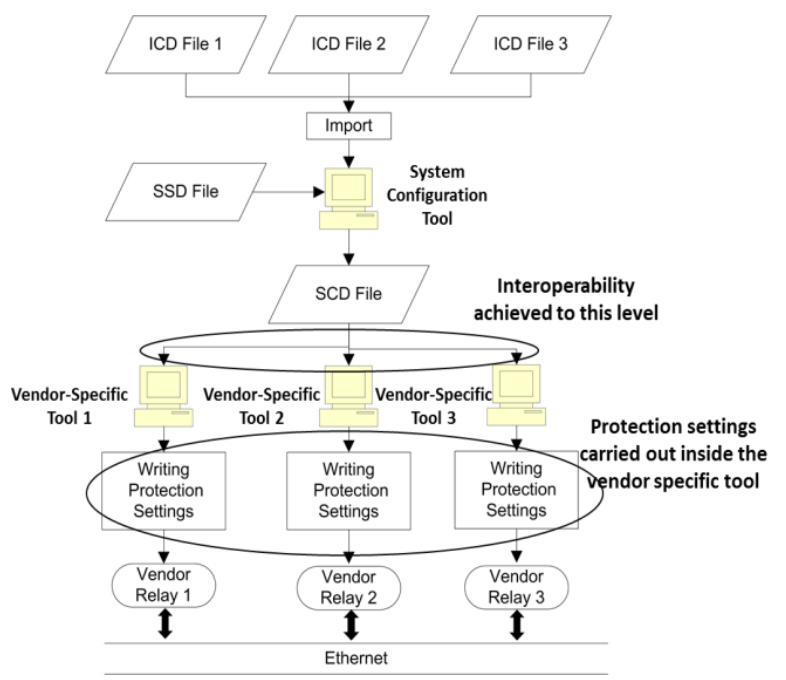

Fig. 9. Existing IED Configuration process [1]

From the view of future power system development, the authors believe full protection setting interoperability offers several compelling benefits, namely:

1. It achieves full interoperability among different vendors" IEDs. Utilities are not "locked-in" to one particular vendor.

2. The configuration process (proposed below) does not need proprietary software or proprietary communications protocols. The engineers only need to be trained to use one tool, which is vendor-neutral.

3. The proposed process is significantly simpler and more streamlined, compared to the existing process.

4. Relay configuration is done at a system level, rather than separately for each vendor/device. This is useful because the protection and control schemes (such as overcurrent protection grading) must also be designed at the system level.

To achieve protection setting interoperability, an IED 61850 configuration process is proposed by the authors as described in the next section.

\section{B. Proposed IED configuration process}

As illustrated in Fig. 10, the proposed IED configuration process includes the following steps:

1. For legacy devices, the original text-based setting files are represented in the IEC 61850 data model through the parsing, mapping and exporting process described in the previous section. For new IEDs which support the proposed process, this step is not needed.

2. The new ICD file is imported into the system configuration tool where the engineers configure the protection settings, if needed, at a system planning level, without considering details about which vendor's product will be used. 
3. The SCD file is imported directly to each IED, although this process is presently not required by IEC 61850 [1].

4. The IEDs retrieve the protection setting information from the SCD file and apply the settings automatically.

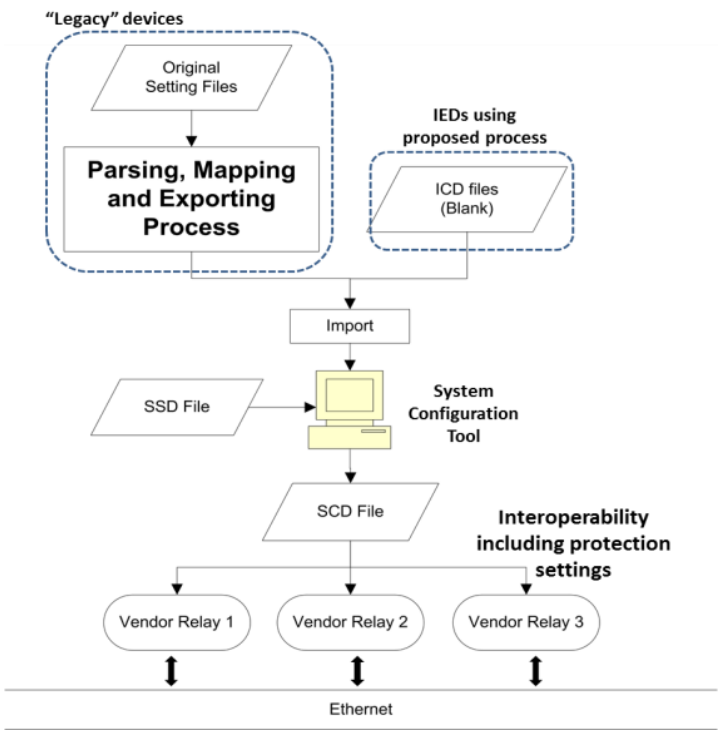

Fig. 10. Proposed IED configuration process

\section{Challenges and possible solutions}

To achieve interoperability of protection settings, there are a number of challenges.

First, the data model in IEC 61850 does not provide a complete set of data objects to describe protection settings for every relay manufacturer. For example, for distance protection, the PDIS class does not provide a data object to describe the shape of the characteristic used (e.g., Mho, Quadrilateral, etc.). Furthermore, the data objects for settings in the PDIS class are all optional. There is no clear definition of when these objects are needed. The standard must be extended to develop a data model which covers all protection setting functions and characteristics.

Second, the data model should be able to be extended in a way that could describe new protection functions in the future, while potentially being understood by multiple devices. One possible solution is that each vendor takes the responsibility for providing a comprehensive description of its new functions (logical nodes). Thus, while the vendors develop new functions, the logical nodes and data objects may keep growing and interoperability would still be maintained for the standardized parts.

Third, IEDs must be developed with the ability to interpret SCD files which contain protection setting information. Furthermore, the proposed approach is best suited for new substations rather than adding to existing substations.

\section{CONCLUSION}

In this paper, the process of representing protection settings in the IEC 61850 data model has been introduced. Through the proposed parsing, mapping, and exporting processes, the original proprietary setting files are converted to well-defined, structured SCL-based setting files, which are then available for multiple smart grid applications. The paper has focused on using SCL-based setting files to achieve protection setting interoperability, and a new IED configuration process has been proposed. This approach brings several significant benefits, such as: achieving full interoperability among IEDs which avoids utilities being "locked-in" to one particular vendor; significantly streamlining the configuration process; no proprietary software or communication protocols are needed; and centralizing the protection setting process to the system level.

Future work will research the required extensions to the IEC 61850 data model, investigate software tools for IEDs to interpret the SCL-based setting files, and carry out case studies for testing interoperability between different IEDs.

\section{REFERENCES}

[1] IEC 61850-6: Configuration description language for communication in electrical substations related to IEDs, IEC Std., 2 ed, 2010.

[2] E. M. Davidson, S. D. J. McArthur, and J. R. McDonald, "A toolset for applying model-based reasoning techniques to diagnostics for power systems protection," IEEE Trans. Power Systems, vol. 18, pp. 680-687, 2003.

[3] Q. Hong, A. Dysko, and C. D. Booth, "Intelligent System for Detecting Hidden Errors in Protection Settings," Universities' Power Engineering Conference (UPEC), London, UK, 2012

[4] IEC 61850-1: Introduction and overview, IEC Std., 1 ed, 2003.

[5] IEC 61850 7-4: Basic communication structure -Compatible logical node classes and data object classes, IEC Std., 2 ed, 2010.

[6] IEC 61850-7-1: Basic communication structure -Principles and models, IEC Std., 2 ed, 2011.

[7] IEC 61850-7-3: Basic communication structure -Common data classes, IEC Std., 2 ed, 2011.

[8] J. E. F. Friedl, Mastering Regular Expressions, California: O'Reilly \& Associates, Inc., 1998, p. 1.

[9] Alstom. (07/11/2012). MiCOMho P443 Technical Manual.

[10] OpenMUC. (25/09/2012). Available: http://www.openmuc.org/index.php?id=24

[11] A. W. McMorran, G. W. Ault, C. Morgan, I. M. Elders, and J. R. McDonald, "A common information model (CIM) toolkit framework implemented in Java," IEEE Trans. Power Systems, vol. 21, pp. 194201, 2006.

[12] NEI Electronic Ltd, "Operating Recommendations THR Distance Protection," pp. 3-4, 1985

[13] EMF. (Eclipse Modeling Framework Project, 26/10/2012). Available: http://www.eclipse.org/modeling/emf/

[14] S. M. Blair, F. Coffele, C. D. Booth, and G. M. Burt, " An Open Platform for Rapid-Prototyping Protection and Control Schemes with IEC 61850," IEEE Trans. Power Delivery, in press.

[15] ENTSO. (07/11/2012). ENTSO-E. Available: http://www.entsoe.eu/fileadmin/user_upload/_library/news/IEC61850_ standard/121018_PunchListTF61850_15102012V4.pdf 